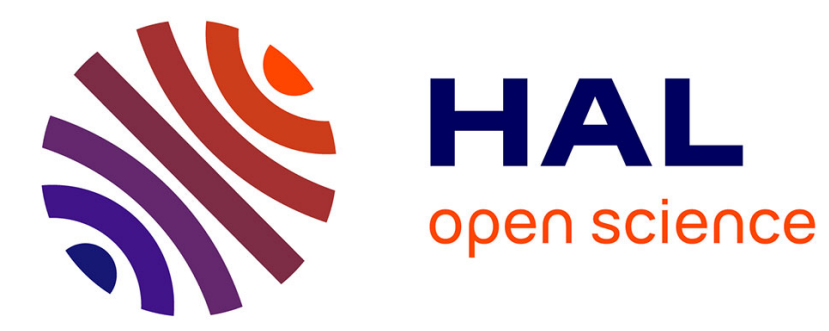

\title{
Des univers ouvriers bousculés par les restructurations répétées
}

Cédric Lomba

\section{To cite this version:}

Cédric Lomba. Des univers ouvriers bousculés par les restructurations répétées. Savoir / Agir , 2017, Les désindustrialisations (re)visitées, 39, pp.57-63. 10.3917/sava.039.0058 . hal-01510255

\section{HAL Id: hal-01510255 \\ https://hal.science/hal-01510255}

Submitted on 19 Apr 2017

HAL is a multi-disciplinary open access archive for the deposit and dissemination of scientific research documents, whether they are published or not. The documents may come from teaching and research institutions in France or abroad, or from public or private research centers.
L'archive ouverte pluridisciplinaire HAL, est destinée au dépôt et à la diffusion de documents scientifiques de niveau recherche, publiés ou non, émanant des établissements d'enseignement et de recherche français ou étrangers, des laboratoires publics ou privés. 


\section{Des univers ouvriers bousculés par les restructurations répétées Cédric Lomba (sociologue, CNRS, CSU-Cresppa)}

Si les restructurations industrielles ont donné lieu à de nombreuses recherches de sciences sociales ainsi qu'à des documentaires ou des œuvres de fiction, elles ont souvent été appréhendées après la fermeture de sites de production pour en saisir les effets dramatiques sur les ouvriers ou sur des espaces régionaux. Pour autant, les fermetures spectaculaires de grandes usines ne constituent qu'une des facettes les plus visibles des restructurations. La fermeture d'une usine n'est bien souvent que l'aboutissement d'un long processus de réorganisations et de plans sociaux partiels. En France, entre 1975 et 1994, les fermetures d'établissements industriels de plus de 100 salariés ont supprimé 600000 emplois tandis que les établissements en activité ont réduit les effectifs de plus d'un million de postes ${ }^{1}$, auxquels s'ajoute la non-reconduction des contrats précaires (notamment des intérimaires). Depuis les années 1970, la plupart des sites industriels ont connu des plans de restructurations, même dans les secteurs en développement $^{2}$, qui se sont traduits par des diminutions d'emplois répétées et des transformations de modes d'organisation du travail et de gestion des travailleurs. C'est en ce sens que la plupart des recherches sur le sujet pensent les restructurations comme un trait constant des économies capitalistes après 1975, et surtout depuis les années 1990, lorsqu'elles ne sont plus seulement défensives (pour éviter une faillite) mais compétitives (pour assurer une position concurrentielle ou pour répondre aux exigences d'actionnaires) ${ }^{3}$. Elles sont devenues plus diffuses, discrètes et continues, ce sont des «actes de la vie ordinaire des entreprises » ${ }^{4}$. Pour autant, en France, mais c'est vrai aussi ailleurs, les experts techniques et les ingénieurs ont vu leur nombre nettement augmenter dans l'industrie, ce sont surtout les ouvriers non qualifiés et dans une moindre mesure les ouvriers qualifiés qui ont subi ces plans de licenciements.

\footnotetext{
${ }^{1}$ Danielle Roualdes, «La restructuration des grands établissements industriels », Insee Première, 1997, $\mathrm{n}^{\circ} 513$.

2 Pierre Fournier, Séverin Muller, Cédric Lomba (dir.), L'industrie pharmaceutique sous observation, Toulouse, Erès, 2014.

${ }^{3}$ Rachel Beaujolin-Bellet, Géraldine Schmidt, Les restructurations d'entreprises, Paris, La Découverte, 2012. ${ }^{4}$ Jacky Fayolle, «Restructurations d'hier et d'aujourd'hui : les apports d'un séminaire ", Revue de l'IRES, $2005, n^{\circ} 1$, p. 341.
} 
On se propose donc d'interroger les rapports qu'entretiennent au travail et à l'ordre usinier ces ouvriers qui ont échappé aux plans de licenciements, mais qui ont subi les effets des réorganisations et les pressions de l'incertitude. Plus spécifiquement, il s'agit de comprendre comment ils vivent ces chevauchements de temporalités, celle à court terme de chaque plan de restructuration qui se présente comme une manière de retrouver la compétitivité de l'entreprise, et celle de moyenne durée, de succession de plans depuis les années 1970. En effet, souvent, les transformations des conditions d'emploi et de travail des ouvriers industriels ne sont ni continues ni brutalement modifiées. Elles relèvent plutôt d'une série d'à-coups successifs.

\section{La planification industrielle au coup par coup}

Pour saisir les logiques gestionnaires à l'œuvre dans l'industrie depuis les années 1970, je m'appuierai sur l'exemple de la sidérurgie belge où j'ai mené des enquêtes lors de deux plans de restructuration (le premier, en 1996-1998, prévoyait de réduire les effectifs et les coûts de 20\% sans fermeture d'usine; le deuxième en 2011-2012 envisageait la fermeture de la moitié des usines). L'entreprise Cockerill était le principal producteur national jusque dans les années 1990, et est devenue après sa privatisation à la fin des années 1990 une sous-division de la branche européenne du premier sidérurgiste mondial (Arcelor puis ArcelorMittal dans les années 2000). Les effectifs de ses usines de l'agglomération de Liège sont passés de près de 25000 personnes en 1975 (20 usines), à moins de 10000 à la fin des années 1980 (11 usines) et moins de 1000 (5 usines) aujourd'hui. Comme pour bon nombre de sites sidérurgiques européens, la baisse des effectifs est quasiment continue sur la période. Les ouvriers de Cockerill, tous des hommes, pour la plupart diplômés de l'enseignement secondaire technique ou professionnel, ont débuté sur des statuts précaires (intérimaires puis CDD pendant plusieurs années) avant d'obtenir un contrat stable et ont réalisé l'intégralité de leur carrière dans un contexte de baisse des effectifs. Pour eux, la restructuration est de fait permanente, mais, jusque 2011, la forte présence syndicale (avec un taux de syndicalisation des ouvriers dépassant les 95\%, un peu plus élevé que dans l'ensemble du pays) permet d'en limiter les effets pour les ouvriers stables, avec l'utilisation quasisystématique des préretraites à partir de 52 ans. Plus généralement, au-delà de cette usine, la plupart des ouvriers industriels européens ont connu un ou plusieurs plans de 
réorganisation des usines dans lesquelles ils travaillent, ils ont pour une part été victimes de licenciements individuels ou collectifs, et ils fréquentent d'autres ouvriers (parents, amis, voisins) qui ont été licenciés. Leur univers cognitif est ainsi traversé du risque de la déstabilisation sociale.

Par ailleurs, l'évolution de la courbe des effectifs de Cockerill donne l'impression d'une dégradation continue à laquelle les salariés pourraient s'adapter ou s'habituer. Ce n'est vrai que dans la mesure où les plans de préretraite étalent les départs sur plusieurs années. En réalité, cette histoire est marquée par des temporalités de moyennes durées et des ruptures saccadées qui compliquent les projections de carrière et de vie. En effet, depuis la fin des années 1970, l'entreprise a connu douze plans de restructurations généraux, et une multitude de plans d'aménagement de l'organisation du travail développés par un grand nombre d'experts, internes ou externes, en gestion et management ${ }^{5}$. Le fonctionnement et la pensée par plans, un dispositif de rationalisation qui peut être élargi à la pratique des managers au $\mathrm{XX}^{\mathrm{e}}$ siècle ${ }^{6}$, ordonne les rythmes de l'entreprise, prescrit et relie différentes dimensions de l'organisation (les investissements, l'organisation du travail, le prix de revient, le volume de l'emploi, etc.). C'est aussi un moyen de critiquer les anciennes pratiques de travail et de mettre en concurrence par le chiffre les hommes et les usines, avec l'usage systématique du benchmarking pour comparer les usines d'ArcelorMittal sur de multiples paramètres. Ces plans produisent un double effet: d'un côté, ils se présentent comme un moyen de résoudre un problème présent (quand l'entreprise connaît des pertes) ou futur (améliorer la compétitivité) laissant augurer un avenir stabilisé pour les ouvriers ; de l'autre côté, l'usage intensif du benchmarking impose un contrôle continu de la performance à toujours renouveler et amplifier. De ce point de vue, l'usage de la planification prend un sens différent dans cette entreprise après les années 1970. Jusque là, la direction, essentiellement composée d'ingénieurs, établissait des plans quinquennaux afin d'organiser les investissements à réaliser. Par la suite, avec les managers, les plans ne sont plus programmés dans une série, chaque plan se présente comme une fin en soi, mais il est de fait suivi d'un nouveau plan dès lors que les mesures du précédent sont appliquées.

\footnotetext{
${ }^{5}$ Cédric Lomba, « Restructurations industrielles : appropriations et expropriations des savoirs ouvriers », Actes de la recherche en sciences sociales, 2013, $\mathrm{n}^{\circ}$ 196-197.

${ }^{6}$ Yves Cohen, Le siècle des chefs. Une histoire transnationale du commandement et de l'autorité (18901940), Paris, Amsterdam, 2013.
} 
$\mathrm{Au}$ final, les ouvriers s'inscrivent, à des degrés divers, dans de multiples temporalités. Ils se sentent à la fois pris dans un mouvement perpétuel de dégradation des conditions d'emploi et sont convaincus de la forte incertitude quant à l'avenir de leur usine. Mais, ils sont également pris dans une temporalité à moyen terme, définie par les plans qui laissent à penser que la situation est difficile mais qu'elle va pouvoir s'améliorer et se stabiliser.

\section{Les carrières mouvementées en temps de crises}

Ces transformations répétées ne sont pas sans conséquences pour les carrières ouvrières. Je ne prendrai qu'un exemple sur le rapport à la carrière au travers de la trajectoire d'un ouvrier à Cockerill. Marco, diplômé de l'enseignement secondaire professionnel, entre comme manutentionnaire à vingt ans dans la sidérurgie en 1985, dans une filiale de Cockerill, après avoir été intérimaire. Comme cette usine connaît beaucoup de chômage partiel (presque quinze jours par mois) durant la crise de 1993, il se trouve un autre poste, plus physique mais aux revenus plus stables, au déchargement de minerais. Cinq ans plus tard, il est déplacé vers le haut fourneau alors que cette usine lui fait peur. Mais il apprend à se passionner pour le métier de fondeur. À l'annonce de la fermeture des hauts fourneaux après la privatisation de l'entreprise, en 2003, il est envoyé à la cokerie où il est mis à l'écart par les jeunes en CDD et les intérimaires qui convoitent son poste, par d'autres ouvriers parce qu'il conserve un salaire élevé et par la maîtrise parce qu'il refuse de travailler sur le poste le plus exposé aux gaz toxiques. Il retourne avec enthousiasme ensuite au haut fourneau que la nouvelle direction d'ArcellorMittal a réouvert pour profiter de la croissance de la demande mondiale d'acier. Il change à nouveau de haut fourneau car le redémarrage est de courte durée alors qu'il était annoncé pour quinze ans, puis il est dirigé vers un poste de pontier dans une autre usine bien qu'il mais il n'aime pas ce travail isolé en cabine. Il revient à nouveau au haut fourneau mais connaît un contexte très difficile car les effectifs ont été entre-temps diminués de moitié et la plupart des ouvriers sont jeunes et, selon lui, sans expérience. Après une nouvelle annonce de fermeture liée à la crise de 2010, il accepte d'être muté dans une usine à plus de 1000 km de là, dans le sud de la France, sans sa femme qui reste dans la ville. Il revient ensuite en Belgique sur un poste peu qualifié dans une usine de galvanisation de Cockerill mais connaît beaucoup de chômage partiel 
(105 jours sur l'année). En 2012, après l'annonce de fermeture définitive de plusieurs usines, dont les hauts fourneaux, il décide de quitter l'entreprise, lassé des changements de postes répétés, des déclassements professionnels, et de la mise à l'écart par ses nouveaux collègues. Il accepte alors d'intégrer une cellule de reclassement mise en place par les pouvoirs publics pour démanteler les usines fermées, mais se présente à ce jour comme « au creux de la vague ».

Cette trajectoire est particulièrement heurtée. D’autres ouvriers, notamment ceux qui travaillent dans des usines moins soumises au chômage partiel ou aux fermetures, présentent des carrières plus stables ou ascendantes pour accéder aux postes d'ouvriers qualifiés ou de petit encadrement. C'est le cas d'ouvriers plus jeunes et plus diplômés qui profitent des départs des anciens en préretraite ou encore des ouvriers plus âgés qui activent des réseaux d'interconnaissance dans l'entreprise pour obtenir les meilleurs postes de reclassement. Il reste que la plupart des ouvriers rencontrés ont connu plusieurs épisodes de changements d'usines ou de service, à leur initiative ou, plus souvent, à celle de l'encadrement. C'est ce que l'on désigne dans l'entreprise comme des «reclassements» mais qui s'apparentent davantage à des « déplacements ». Ce qui pourrait être vu de loin comme des aménagements de postes sans changement de qualification constitue pour eux de véritables mobilités sociales et professionnelles. Ces déplacements impliquent en effet des formes d'adaptations à un poste ou un métier (il y a peu en commun entre les tâches de fondeur, de pontier ou d'opérateur à la cokerie), à un groupe de travail, à un univers organisationnel, et pour certains à un collectif militant. La trajectoire de Marco donne aussi à voir le rapport subjectif à l'emploi, à l'usine et au travail qui passe par des phases d'anticipations, parfois réussies mais souvent avortées, sans linéarité claire. Pour reprendre les distinctions établies par Serge Paugam7 ${ }^{7}$, ces travailleurs passent, parfois dans des temps très courts, d'une intégration assurée à une intégration laborieuse voire incertaine. Et tout laisse à penser que l'on observe depuis les années 1970 un renforcement de l'incertitude professionnelle, même au sein du pôle le plus stable du monde ouvrier.

\footnotetext{
${ }^{7}$ Serge Paugam, Le salarié de la précarité. Les nouvelles formes de l'intégration professionnelle, Paris, Presses Universitaires de France, 2000.
} 


\section{Les rapports des ouvriers au travail et à l'entreprise par gros temps}

Mener sa vie professionnelle dans un tel contexte d'incertitude produit des effets sur les rapports qu'entretiennent les ouvriers à leur travail et plus largement sur leurs représentations du monde social. Certains sont particulièrement sensibles au risque de fermeture, notamment ceux qui occupent des positions basses dans la hiérarchie ouvrière. C'est le cas de Robert du service Traction, né au début des années 1960, diplômé de l'enseignement secondaire inférieur et issu du bas des classes populaires (sa mère vivait de petits boulots de service et son père a abandonné le foyer quand il était enfant). Évoquant les plans de restructurations, Robert précise :

« Il y a des gens indésirables qu'on a envoyé au four à coke. Par exemple, on était quarante et il fallait en sortir dix. Et à chaque fois on se regardait en chien de faïence. Il y en a qui ont pas eu le choix. Il y avait de la peur. On a toujours travaillé avec la peur au ventre, d'être déplacé et maintenant de perdre son emploi. On a jamais jeté personne dehors, mais de très peu. Maintenant, je suis le plus vieux, mais ça ne veut rien dire, on ne parle plus d'ancienneté, ils parlent de capacités ».

D'autres sont plus sereins sur l'avenir industriel et leur propre carrière. Ainsi, c'est plutôt l'étonnement qui prévaut parmi les ouvriers lors de l'annonce de fermeture de la moitié des usines de Cockerill en 2011, alors que la menace de restructuration plane en permanence, comme un danger sourd, depuis plusieurs décennies. Plus encore, une partie se montre blasée, comme cet ouvrier diplômé de l'enseignement secondaire supérieur embauché dans les années 1990 :

«Depuis que j'ai commencé, on m'a toujours dit "On va fermer et tu vas voir en telle année on va fermer". On en parle tellement qu'on se dit que ça n'arrivera jamais. J'espère que comme les autres fois une solution va être trouvée ».

À force d'être répétées, les menaces opèrent moins, et ce d'autant que les mobilisations collectives permettent, jusque-là, d'éviter les licenciements au profit des préretraites. Dans le même temps, ces ouvriers sont aux aguets, à la recherche de signes qui pourraient garantir la pérennité de l'usine ou au contraire son arrêt : l'attention se porte sur les mutations des cadres, sur les investissements dans l'usine ou dans les autres sites du groupe, etc. Beaucoup sont convaincus qu'ils ont fait suffisamment d'efforts et de sacrifices en acceptant les diminutions d'effectifs antérieures et que l'on est arrivé au bout du processus. Les moments de relance, associés aux plans, sont aussi 
des périodes de réenchantement. Ainsi, la fermeture temporaire de 2009 intervient après une période de relance que plusieurs ouvriers qualifient d'euphorique, comme l'évoque cet ouvrier qualifié de l'aciérie :

« Les derniers jours de production ont été intensifs avec battages de record. [...]. On travaillait très bien puisqu'avec la moitié de personnel, on fait plus de tonnages qu'avant [...]. Tout le monde était content. Avec la qualité, ça tournait, mis à part un ou deux incidents. Il faut quand même vous dire qu'on a relancé ce bazar-là sans moyens financiers. C'était presqu'un miracle de la relancer [...]. Bref on était sur un nuage, on se disait on va y aller. Moi aussi, je me disais fin de carrière, on va souffler, c'est fini. La crise est arrivée et du jour au lendemain, on nous disait bravo et le lendemain on ferme. »

Si ce contexte d'ensemble touche tous les ouvriers, ils n'y réagissent pas de la même manière. On peut ainsi distinguer des figures d'attachements ou de rejets à l'entreprise ou au travail. Certains, peu nombreux, affichent une loyauté envers l'entreprise. Ils ne mettent presque jamais en cause la direction, justifient les réductions d'effectifs, et critiquent les pouvoirs publics, les syndicats et les travailleurs désinvestis. Ces ouvriers consentants, souvent d'origine ouvrière et plutôt conformistes d'un point de vue moral, ont connu une mobilité professionnelle ascendante. D'autres, plus nombreux, critiquent plus ou moins publiquement les décisions de la direction et l'ordre établi dans les usines. Ils le font au nom d'une perte de reconnaissance des savoir-faire de métier, ou de la détérioration des collectifs ouvriers, ou de la dégradation des conditions matérielles d'exercice, ou encore de l'intensification du travail. Ce sont là des registres de la critique, portés par différents ouvriers, qui participent à lire les rapports au travail comme des rapports de classe. Lorsque Marco met en avant le savoir-faire technique des fondeurs, il insiste sur la dimension collective de l'activité dans un contexte de travail à la fois fascinant et éprouvant. Il détaille l'entraide entre fondeurs et l'ambiance conviviale. D'autres partagent cet attrait du travail, mais ils le vivent de manière plus isolée, plutôt contre les autres qu'avec eux, en défendant la préservation de leur savoir-faire technique négligé par l'orientation financière des firmes ${ }^{8}$. Plus généralement, le rapport instrumental au travail des ouvriers aux salaires élevés de Luton étudiés par John Goldthorpe et son équipe dans les années $1960^{9}$, ne constitue à Cockerill qu'un des rapports possibles au travail. Ce rapport instrumental coexiste avec

\footnotetext{
${ }^{8}$ Cf. Massimiliano Mollona, Made in Sheffield: An Ethnography of Industrial Work and Practice, New York and Oxford, Berghahn Books, 2009.

9 John H. Goldthorpe and al., L'ouvrier de l'abondance, Paris, Seuil, 1972.
} 
d'autres formes de représentations subjectives et d'autres modes d'engagement au travail (« solidaristes », défense du métier, etc.).

Surtout, avec les basculements de carrière inopinés comme ceux qu'a subi Marco, ces postures sont susceptibles d'évoluer non pas seulement selon les périodes, par exemple avec le passage d'une orientation solidariste à une orientation instrumentale, mais selon les trajectoires sociales et professionnelles des ouvriers en contexte. Des déplacements répétés, des blocages de carrière, ou au contraire des opportunités de promotion suite aux départs des anciens, peuvent modifier en profondeur ce rapport à l'usine. Prendre en compte ces temporalités et trajectoires aide à comprendre les représentations professionnelles et sociales des ouvriers, ainsi que leurs engagements. De même que les effets de chaque plan réactivent la critique de la dégradation des conditions d'emplois et de travail, il réenchante momentanément les représentations d'une partie de ceux qui restent. Toutefois, comme la rationalité de la succession des plans est peu évidente, la production d'un contre-discours argumenté est délicate. Dans le cas de Cockerill, si les organisations syndicales ont longtemps proposé des solutions alternatives à celles des dirigeants financiers et des consultants, les interprétations se brouillent en temps de crises répétées et ce d'autant que les plans antérieurs ont largement déstructuré l'organisation productive. Les dernières années, les critiques se personnalisent sur l'enrichissement ou la culture ethnique d'un PDG éloigné -Lakshmi Mittal-, et des ouvriers mettent en cause des leviers de pouvoir qu'ils peuvent atteindre comme les directions syndicales ou les pouvoirs publics wallons accusés d'avoir vendu l'entreprise au privé ou de ne pas nationaliser. Du coup, la critique délaisse en partie les problématiques de l'ordre usinier et peine à envisager des agencements industriels qui ne répondent pas aux exigences de rentabilité à court terme du capitalisme financier. 